\title{
Transgenic Mouse Studies to Understand the Regulation, Expression and Function of the Testis-Specific Protein Y-Encoded (TSPY) Gene
}

\section{Stephanie Schubert* and Jörg Schmidtke}

Institute of Human Genetics, Hannover Medical School, D-30625 Hannover, Germany;

E-Mail: schmidtke.joerg@mh-hannover.de

* Author to whom correspondence should be addressed; E-Mail: schubert.steffi@ mh-hannover.de; Tel.: +49-511-532-3874; Fax: +49-511-532-5865.

Received: 30 June 2010; in revised form: 13 August 2010 / Accepted: 16 August 2010 /

Published: 18 August 2010

\begin{abstract}
The TSPY gene, which encodes the testis-specific protein, Y-encoded, was first discovered and characterized in humans, but orthologous genes were subsequently identified on the Y chromosome of many other placental mammals. TSPY is expressed in the testis and to a much lesser extent in the prostate gland, and it is assumed that TSPY serves function in spermatogonial proliferation and/or differentiation. It is further supposed that TSPY is involved in male infertility and exerts oncogenic effects in gonadal and prostate tumor formation. As a member of the TSPY/SET/NAP protein family, TSPY is able to bind cyclin B types, and stimulates the cyclin B1-CDK1 kinase activity, thereby accelerating the $\mathrm{G}_{2} / \mathrm{M}$ phase transition of the cell cycle of target cells. Because the laboratory mouse carries only a nonfunctional Y-chromosomal Tspy-ps pseudogene, a knockout mouse model for functional research analyses is not a feasible approach. In the last decade, three classical transgenic mouse models have been developed to contribute to our understanding of TSPY regulation, expression and function. The different transgenic mouse approaches and their relevance for studying TSPY regulation, expression and function are discussed in this review.
\end{abstract}


Keywords: testis-specific protein, Y-encoded (TSPY); TSPY-like proteins; male meiosis; Gonadoblastoma oncogene; germ cell tumor stem cells; cell cycle regulation; transcription regulation; neural functions; tumor suppressor; sexual dimorphisms

\section{Introduction}

The human TSPY cluster on the human Y chromosome is unique because it represents the largest and most homogenous protein-coding tandem array in the human genome [1]. The major cluster is located close to the centromere on Yp11.2, where each $2.8 \mathrm{~kb}$ TSPY copy is embedded in a single $20.4 \mathrm{~kb}$ DYZ5 tandem repeat array unit [2,3]. While many MSY genes degenerate during evolution due to accumulation of deleterious mutations as a consequence of their suppressed recombination with their X-homologs [4], others avoid degeneration by being present in multiple, nearly identical copies, thereby having the chance to compensate the effects of null alleles via unequal sister chromatid exchange or Y-Y gene conversion [1,5]. This strategy is also applied to the TSPY gene, being organized in multiple copies and functionally conserved in primates and cattle [6-9]. In healthy men, $T S P Y$ copy number can vary between individuals, ranging from 23 to 64 copies per genome, including functional members and pseudogenes [10]. Functional TSPY copies can differ from one another by up to $1 \%$ in coding sequences and promoter regions [5].

The Tspy organization in murid rodents is quite different, in that only single-copy Tspy-ps pseudogenes exist on the $\mathrm{Y}$ chromosome in species of the subgenus Mus (including the laboratory mouse) [11,12], and in the Mongolian gerbil [13], while several Apodemus species and the rat possess only one functional Tspy gene. Similar to the human and bovine situation, repetitive functional Tspy genes persist on the Y chromosomes of Mus platythrix and the Syrian hamster [12,13].

In humans, TSPY is mainly expressed in fetal and adult testis, where its expression is limited to gonocytes and prespermatogonia during fetal and neonatal development, and restricted to spermatogonia, primary spermatocytes and round spermatids in adult testis [14-16]. TSPY is also expressed, although much more weakly, in epithelial cells of the prostate gland [17], and some TSPY ESTs derived from the medulla of human brains (BX281192, B1828033) have also been reported in databases [16]. Recently, TSPY has been classified as a tissue-enriched gene that is highly expressed in a specific organ (testis) and is not expressed, or is expressed at much lower levels, in other tissues [18]. Northern blot and RT-PCR analyses have shown that TSPY is not transcribed in human organs such as heart, lung, liver, spleen, small intestine, kidney, thymus and in leukocytes, however other tissues have not been analyzed up to now $[7,19,20]$.

TSPY expression pattern within the testis as well as its homology to other cyclin B binding TSPY/SET/NAP protein family members point to a role in germ cell proliferation and/or meiotic differentiation. What diverse function(s) TSPY may fulfill in spermatogonial proliferation, differentiation and male meiosis within the testis, and whether it also has a physiological function in the prostate, is currently unknown. It was shown in stably transfected human HeLa and mouse NIH3T3 cells that TSPY could mediate proliferative properties by enhancing the $\mathrm{G}_{2} / \mathrm{M}$-phase transition of the cell cycle [21]. This effect is directly mediated by the binding of TSPY to the cyclin 
B1-CDK1 complex, and enhancing its kinase activity [22]. A yeast-two hybrid approach recently identified the eukaryotic translation elongation factor 1 alpha (eEF1A), which is essential for the elongation phase during protein translation, as a second binding partner for TSPY [23]. The findings of the latter study point to a possible function of TSPY in gene transcription and protein translation by interaction with eEF1A.

TSPY expression is up-regulated in testicular seminoma [24], some non-seminomatous germ cell tumors [25], carcinoma-in situ (CIS) [15,26] and gonadoblastoma [27-29], and the TSPY gene is regarded as the candidate for $G B Y$, the elusive gonadoblastoma locus on the human Y chromosome that is thought to have a normal function in the testis but to act as an oncogene in the dysgenetic gonad of XY-sex-reversed and intersex humans [30]. TSPY is also strongly expressed in some somatic tumors, including prostatic carcinoma, hepatocellular carcinoma and melanoma [17,31,32]. However, up to now there is no evidence for a causative role of TSPY in gonadal or somatic tumorigenesis.

Transgenic mice are a powerful tool for promoter, expression and functional research analyses of a specific gene in vivo, and since the first use of the classical pronuclear injection technique in 1980, thousands of transgenic mouse lines have been generated [33,34]. The evolutionary knock-out of the Tspy-ps in species of the subgenus Mus, including the laboratory mouse [11,12,35], has enabled the generation of different transgenic mouse models for studying human TSPY regulation, expression and function in vivo.

\section{TSPY transgenic mouse models}

2.1. Transgenic mouse line TSPY-TAg23: SV40 large and small TAg under the control of a $1.3 \mathrm{~kb}$ putative TSPY promoter fragment

2.1.1. Generation of TSPY-TAg transgenic mice to investigate the susceptibility of spermatogonia to malignant transformation

Many studies have used transgenic mouse models expressing the viral oncogene simian virus 40 (SV40) large tumor antigen (TAg) under the control of a tissue- or cell-specific promoter for targeted tumor formation, in order to generate suitable animal models for specific types of cancer that can be used to investigate the molecular mechanisms in cellular transformation and evaluate new therapies in oncogenesis [36,37]. It is well known that large tumor antigen TAg encoded by SV40 contributes to cell transformation, in part by binding to and blocking the functions of the tumor suppressors $\mathrm{pRb}$ and p53 [38,39].

The ability to express SV40 TAg in specific cell types by the use of a cell-specific promoter could be a powerful tool to determine the susceptibility of testicular germ cells to transformation by SV40 $\mathrm{TAg}$ at various stages of differentiation. However, this strategy is also limited by some facts. Mice are not commonly susceptible to testicular type-II germ cell tumors (seminomatous, nonseminomatous tumors) and to spermatocytic seminomas, and only testicular teratomas arise spontaneously in mice of the strain 129 with an incidence of 1-8\%, depending on the substrain [40-42]. Although it is commonly supposed that a delayed or blocked maturation of a primitive germ cell, such as a primordial germ cell (PGC) or gonocyte could be the initial step that leads to formation of most testicular germ cell tumors, the cellular origin of the bulk of testicular germ cell tumors is still unknown [40]. Therefore, 
generation of transgenic mice in a defined genetic background expressing TAg in early male germ cells such as PGCs or gonocytes could offer useful information about the cellular origin of testicular germ cell tumors, and uncover the molecular mechanisms that lead to tumor formation.

The first TSPY transgenic mouse line (TSPY-TAg23) was published in 2003, and was generated in an attempt to investigate the susceptibility of mouse spermatogonia to malignant transformation [43]. This transgenic line was founded on fertilized one cell embryos of FVB mice and was produced using the viral oncoprotein simian virus 40 (SV40) large and small $\mathrm{T}$ antigen (TAg), driven by a $1.3 \mathrm{~kb}$ putative human $T S P Y$ promoter fragment. Five founder transgenic mice were generated via pronucleus injection from which only one male (TSPY-TAg23) could be out-crossed with FVB wild-type mice and transmitted the transgene to its progeny, thereby founded the transgenic line TSPY-TAg23.

2.1.2. $1.3 \mathrm{~kb}$ of a $T S P Y$ promoter is insufficient to direct a proper tissue-specific expression pattern of $\mathrm{TAg}$ in transgenic mice

In 2003, it was still believed that TSPY expression is strictly testis-specific. TSPY transcripts had been detected in testes of 22 and 26 week old human fetuses [7], and immunohistochemical analyses of adult human testes localized TSPY predominantly to spermatogonia and to a much weaker extent to primary spermatocytes [15]. The regulation of TSPY was still unknown, and only $1.2 \mathrm{~kb}$ immediately $5^{\prime}$ to the start of TSPY gene transcription had been identified as a potential promoter region that precedes all or almost all TSPY genes [5]. Tascou et al. [43] used $1.3 \mathrm{~kb} 5$ 'flanking region of a functional TSPY gene in an approach to express the SV40 TAg oncogene in early testicular germ cells. This fragment was initially analyzed in vitro in transiently transfected mouse spermatogonia derived GC-1 spg and mouse spermatocyte (between preleptotene and early pachytene stage) derived GC-4spc cell lines [44], and was shown to induce a higher transcriptional activity of a chloramphenicol transferase (CAT) reporter gene in GC-1spg cells in comparison to GC-4spc cells. This study was the first attempt to test a putative TSPY promoter fragment in transgenic mice in vivo.

The authors were able to detect TAg transcripts via RT-PCR analyses not exclusively in the testis but also in the spleen, seminal vesicle, pituitary gland and adrenal gland of transgenic mice (Table 1).

Thus, it seems that $1.3 \mathrm{~kb} 5^{\prime}$-region of a TSPY gene is insufficient in mediating a proper tissuespecific expression pattern in transgenic mice. However neither data about the transcription level nor about a proper translation of the transgene in the respective TAg expressing transgenic organs and tumors were reported [43]. Furthermore, no findings about the cellular testicular expression pattern of SV40 TAg driven by the used TSPY promoter fragment were published.

2.1.3. Expression of TAg failed to induce testicular germ cell tumors in transgenic mice but led to tumor formation in pituitary and adrenal glands and the seminal vesicles

The transgenic mouse line TSPY-TAg23 was originally generated to prove the potential of early male germ cells (spermatogonia) for malignant transformation, via targeted expression of SV40 large TAg, but large tumor antigen TAg failed to induce testicular germ cell tumors in transgenic mice [43]. Spermatogenesis was normal in TSPY-TAg23 transgenic mice and neither TSPY-TAg transgenic founders nor male offspring of the line TSPY-TAg23 developed testicular tumors [43]. This is probably 
due to the insusceptibility of mice for most testicular germ cell tumors, and the inherent strain differences in susceptibility.

It is noteworthy that noninvasive pituitary adenomas that were originating from the anterior lobe occurred in approximately $65 \%$ of male and female offspring of the line TSPY-TAg23, and that also three other founder animals containing the TSPY-TAg transgene in their genome developed such tumors [43]. Approximately $20 \%$ of pituitary tumor cells expressed prolactin (PRL), while others expressed the adrenocorticotropic hormone (ACTH). Plasma PRL and corticosterone concentration were significantly increased in TSPY-TAg transgenic male and female mice in comparison to wild-type FVB mice. The strategy to induce targeted tumorigenesis by expression of SV40 large TAg under the control of $1.3 \mathrm{~kb}$ flanking sequences of the human TSPY gene led by the way to the generation of the first available mouse model for analyses of pituitary adenomas containing both, PRL and ACTH secreting tumor cells $[43,45]$. $31 \%$ of all mice with pituitary tumors (transgenic mice of the line $T S P Y-T A g 23$ and one male TSPY-TAg transgenic founder) developed additionally tumors originating in the adrenal medulla. Tumorigenesis occurred also in a few older male progeny of founder 23 that developed a tumor of the seminal vesicle. All the different tumors derived independently due to the ectopic TAg expression in the respective organs.

Table 1. Tissue specific expression pattern of mouse TSPY-ps, and the transgenes in the transgenic lines TSPY-TAg23, Tg(TSPY-cre)33aYfcl and Tg(TSPY)9 Jshm.

\begin{tabular}{|c|c|c|c|c|}
\hline $\begin{array}{l}\text { Expression } \\
\text { analyses in: }\end{array}$ & $\begin{array}{c}1.3 \mathrm{~kb} \text { TSPY promoter } \\
(\text { TSPY-TAg23) }\end{array}$ & $\begin{array}{c}2.4 \mathrm{~kb} T S P Y \text { promoter } \\
T g(T S P Y \text {-cre }) 33 a Y f c l\end{array}$ & $\begin{array}{c}2.9 \mathrm{~kb} T S P Y \text { promoter } \\
T g(T S P Y) 9 \mathrm{Jshm} \\
\end{array}$ & $\begin{array}{c}\text { mouse } \\
\text { TSPY-ps }\end{array}$ \\
\hline brain & - & $+/ \#$ & $+/++$ & + \\
\hline pituitary gland & + & \#* & n.a. & n.a. \\
\hline testis & + & $+/ \#$ & $+/++/ \#$ & + \\
\hline prostate & n.a. & - & $+/--$ & - \\
\hline seminal vesicle & + & - & n.a. & - \\
\hline epididymis & n.a. & n.a. & $+/--$ & n.a. \\
\hline ovary & - & $+/ \#$ & n.a. & n.a. \\
\hline uterus & n.a. & - & n.a. & n.a. \\
\hline lung & - & - & $+/--$ & + \\
\hline heart & - & - & $+/--$ & + \\
\hline spleen & + & + & $+/--$ & + \\
\hline liver & - & - & $-/--$ & + \\
\hline kidney & - & - & + & + \\
\hline adrenal gland & + & n.a. & n.a. & n.a. \\
\hline stomach & n.a. & n.a. & -- & n.a. \\
\hline large intestine & n.a. & n.a. & -- & n.a. \\
\hline thymus & n.a. & - & n.a. & + \\
\hline
\end{tabular}

n.a. not analyzed, detection of transgenic transcript via RT-PCR analyses (+) or Northern blot analyses $(++)$, transcripts absent in RT-PCR analyses (-) or Northern blot analyses (--), transgene could be detected by immunostaining or expression of EGFP (\#), no EGFP expression observed (\#*). Data adapted from [16,43,47]. 
As several founders with different chromosomal integration sites of the transgene developed pituitary adenomas and adrenal medulla tumors it is unlikely that TAg expression in both glands is caused by a chromosomal position effect on transgene expression [46]. The expression of SV40 large and small $\mathrm{T}$ antigen (TAg) in both organs seems to be rather mediated by the used TSPY promoter fragment which directed an ectopic expression of TAg also in other organs than the testis. It was later shown that the $2.4 \mathrm{~kb} 5$ 'region of a TSPY gene is not capable of inducing a Cre-recombinase reporter gene transcription in seminal vesicles of male Tg(TSPY-cre)33aYfcl and Tg(TSPY-cre)33bYfcl transgenic mice, and that $2.4 \mathrm{~kb}$ of the $T S P Y$ promoter is insufficient to induce a reporter gene expression (EGFP) in the anterior lobe of the pituitary gland in double transgenic TSPY-Cre;Z/EG mice (Table1; [16]). Especially the findings of the latter study clarified the inability of the $1.3 \mathrm{~kb} T S P Y$ promoter fragment used to induce a proper tissue-specific expression pattern in transgenic mice. However, it cannot be ruled out that TSPY is also ectopically expressed in organs other than testis and prostate gland. The TSPY gene was recently classified as a tissue-enriched gene that is expressed at a much higher level in a specific organ (testis) than in other tissues [18], and further studies are needed to clarify the tissue-specific expression pattern of human TSPY.

\subsection{The TSPY transgenic mouse line $\mathrm{Tg}(\mathrm{TSPY}) 9 \mathrm{~J}$ shm carrying a functional human TSPY gene}

2.2.1. The TSPY transgenic mouse line $T g(T S P Y) 9 J s h m$, an animal model closely resembles the organization and expression of human TSPY

The putative oncogenic role of human TSPY in dysgenetic gonads and in testicular and prostatic tumor formation and also its function during fetal and adult male germ cell development could be much easier uncovered by the generation of a suitable animal model. With this in mind, repeated efforts finally culminated in only a single $T S P Y$ transgenic mouse line ( $T g(T S P Y) 9 J \operatorname{shm})$ that was successfully established in 2003 by our group [47]. An $8.2 \mathrm{~kb}$ genomic fragment, containing a complete functional human TSPY gene (2796 bp), 2923 bp putative TSPY promoter region and $2481 \mathrm{bp}$ 3' region of human TSPY that was originally derived from the human cosmid cEMT [2] was microinjected in male pronuclei of fertilized oocytes of the outbred strain NMRI and led to the generation of 3 founding transgenic mice (two females and one male). We initially showed that this genomic fragment was correctly transcribed and spliced according to the human pattern in transiently transfected mouse spermatogonial derived GC-1 spg cells [48]. Although all founders were fertile, only the male founder (M9) transmitted the transgene to its progeny with paternal inheritance pattern. FISH and Southern blot analyses showed that approximately 50 copies of the human transgene are clustered at a single site at the distal part of the long arm of the mouse $\mathrm{Y}$ chromosome in the line $\operatorname{Tg}($ TSPY)9Jshm (Figure 1).

The similarity to the human TSPY organization in this transgenic line is a striking feature [2,3,5,49]. The transgene is primarily expressed in the testes, and mostly spliced in accordance with the human pattern [47]. In humans a TSPY transcription unit is $2.8 \mathrm{~kb}$ in length, is composed of 6 exons and 5 introns and generates a $1.3 \mathrm{~kb}$ main transcript, that codes for a polypeptide of 308 amino acids [5,17]. $T S P Y$ transcripts originating from the same TSPY transcription units are frequently alternatively spliced thereby producing various TSPY isoforms of yet unknown function. Up to now, 11 different $T S P Y$ splice variants that vary in sequence and length have been identified in prostatic, testicular and 
testicular tumor tissues $[3,7,15,17,26]$ and many of these are also present in the testes of TSPY transgenic mice (Figure 2; [47,50,51]).

Figure 1. Fluorescence In Situ Hybridization analysis of a biotin-deoxyuridine 5-triphophate-labeled human TSPY probe (pHerbi) to DAPI counterstained chromosomes (A, B) of a TSPY transgenic adult mouse of the transgenic line Tg(TSPY)9Jshm. The recombinant plasmid pHerbi contains the human $8.2 \mathrm{~kb}$ genomic TSPY fragment that was used to generate the TSPY transgenic line Tg(TSPY)9Jshm [47]. Figure 1B was originally published in Schubert et al., 2003 [47].
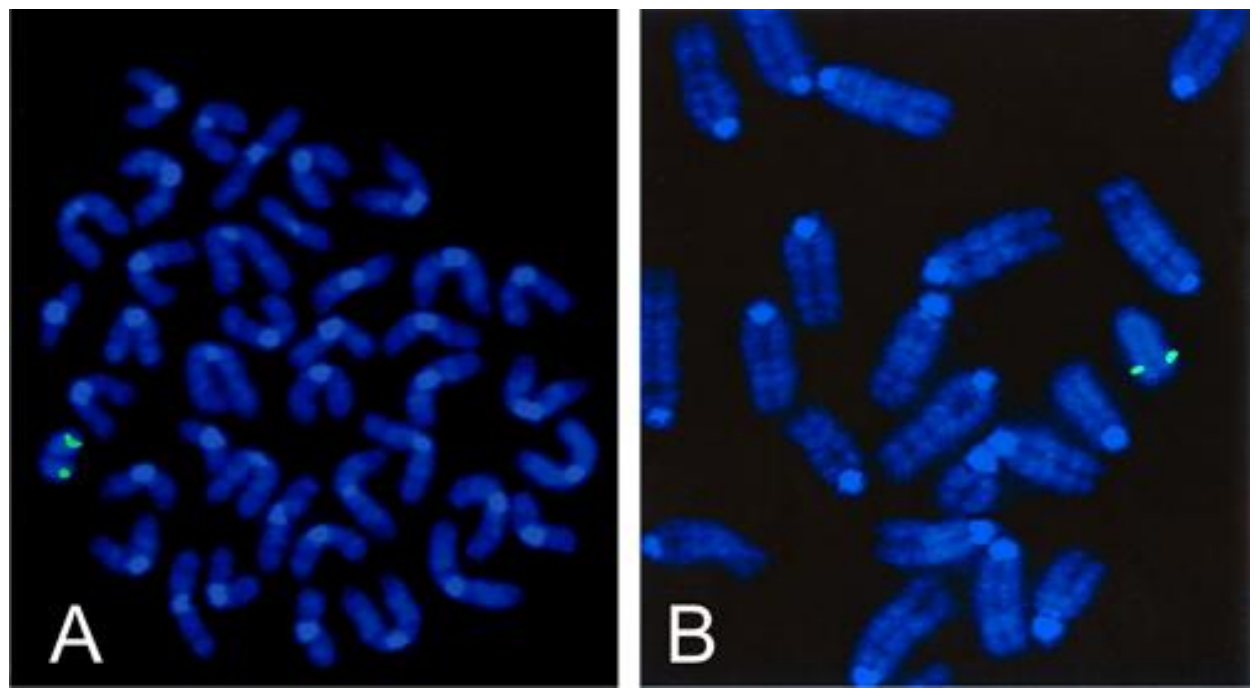

All of these encode polymorphic TSPY isoforms harboring either the whole or part of the cyclin B binding SET/NAP domain of TSPY at their carboxyl termini [47,50-52]. Thus, this model can be useful in further studies for functional analyses of the different TSPY isoforms. It is yet uncertain whether the various different human TSPY transcripts originate from one or more specific or from all functional human TSPY transcriptional units, but this mouse model clearly demonstrates that many $T S P Y$ transcripts can be generated by one particular functional (though repetitive) TSPY transcription unit via alternative splicing.

Northern blot analyses of different male organs (testis, epididymis, prostate, brain, lung, heart, stomach, large intestine, liver, spleen) showed that TSPY is expressed only in the testis and to a much lesser extent in the brain of adult transgenic males [47]. Using RT-PCR, the transgene has been shown to be transcribed at basal levels in other organs, such as the epididymis, prostate, kidney, lung, heart and spleen (Table1; [52]). Hence also 2923 bp 5'-flanking region of the TSPY gene are insufficient to induce a strictly testis and prostate specific expression pattern in transgenic mice. It was shown that the mouse Tspy-ps pseudogene, although being non-functional, is transcribed most prominently in the testis, but also at lower levels in other organs, such as the brain, thymus, lung, heart, liver, spleen and kidney (Table 1; [16]). Thus, the TSPY transgenic expression pattern mimics partially that of the murine counterpart. It is also conceivable that trans-acting factors mediating a proper TSPY expression are less conserved in human and mice. Although the tissue specific expression pattern of the human transgene does not exactly reflect the human pattern, the TSPY transgene is properly regulated within the fetal, neonatal and adult testis [47,52]. As in humans, the TSPY transgene is mainly expressed in 
spermatogonia and in primary spermatocytes at the preleptotene, leptotene, and zygotene stage in postnatal testes [47,50-53], and in gonocytes and prespermatogonia in fetal testes (Figure 3; [14]).

Figure 2. Schematic illustration of a human TSPY gene and the various TSPY transcripts in human prostatic (P, prostatic adenoma and benign prostatic hyperplasia) and testicular (T, human testis; TC, human testicular seminoma) tissues [17] and in mouse testes of the transgenic line $\mathrm{Tg}(\mathrm{TSPY}) 9 \mathrm{~J} \mathrm{shm}$ (TG) [47,50,51]. Introns are shown as grey bars and exons indicated as colored boxes. Small grey bars above the splice variants indicate the use of alternative splice donor and/or acceptor sites. The TSPY cDNA region encoding the cyclin B binding domain (SET/NAP domain) is highlighted by a black line (aa residues 121-265) [22]. * [52]. Detailed descriptions of the differential spliced TSPY transcripts are given in reference [17]. Splice variants are termed in accordance to references [17] and [26]. $18 \mathrm{bp}$ insertion: in frame insertion of GTG GAG CTG GTG GCG CAG within exon 1 of human TSPY, +: combination of different splicing patterns.

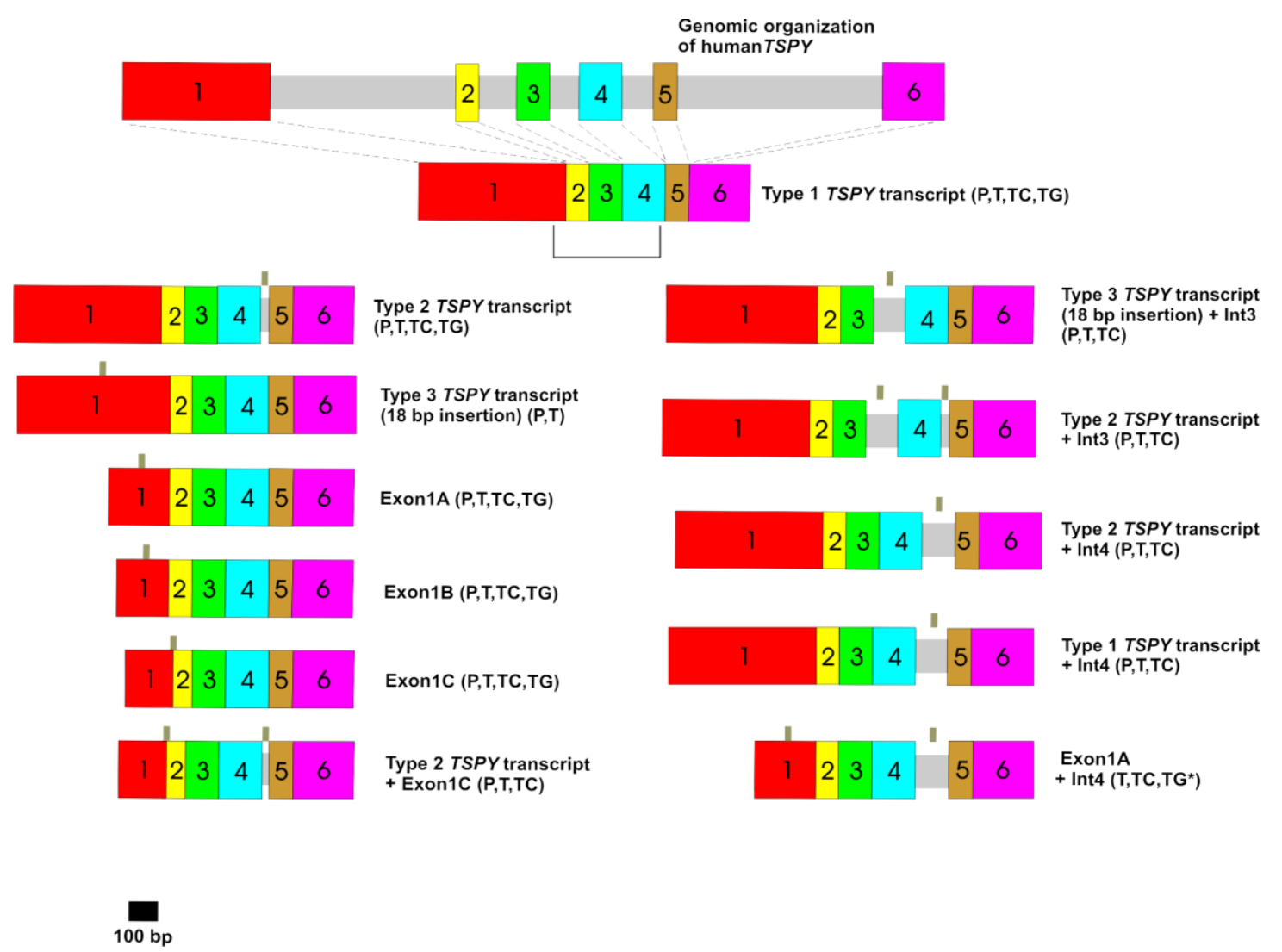

The testicular expression pattern and the cellular topology of the TSPY transgene within testes of the different ontogenetic stages seem to be nearly in total accordance with the human situation. These observations suggest that the line $T g(T S P Y) 9 J$ shm could be useful for further analyses of the regulation and expression of human TSPY.

Initial studies demonstrate that TSPY transcription levels can be raised by androgene administration in androgen-responsive human prostate cancer cell line LNCaP, suggesting that TSPY expression could be regulated by androgens and its receptor within the testis and the prostate [54,55]. Nonetheless, another study has shown that TSPY transcription is upregulated in androgen-independent 
LNCaP-C81 prostate cancer cells in comparison to androgen-dependent LNCaP-C33 cells [56]. As androgens play an important role in the development and differentiation of the human prostate and in prostatic tumorigenesis, and TSPY expression is elevated in prostate cancers of increasing Gleason grades, one may speculate that TSPY exerts an oncogenic or tumor predisposition role in prostate cancer formation [17,55]. If this is mediated by an aberrant and/or androgen-responsive TSPY expression in epithelial cell of the native prostate and/or tumor cells of prostate cancers remain to be elucidated. As the human TSPY transgene is also expressed and correctly spliced in the prostate of transgenic mice of the line $T g(T S P Y) 9 J s h m$ [52], this mouse model could be also useful to address these issues.

Figure 3. Testicular histology of fetal and adult TSPY transgenic mice (Tg(TSPY)9Jshm) and of an adult NMRI wildtype male. Hematoxylin and eosin staining of testis of an adult TSPY transgenic mouse (A) in comparison to an adult NMRI wildtype male (B). Spermatogenesis and spermiogenesis of TSPY transgenic males were normal in comparison to wildtype mice. The TSPY specific antiserum 837/3 [15] immunostained spermatogonia and primary spermatocytes of the leptotene/zygotene stage in adult testes (C) and gonocytes and prespermatogonia in fetal gonads ( $\mathbf{D}$, testis of the embryonal stage $14.5 \mathrm{dpc}$ ) of mice of the line $T g(T S P Y) 9 J s h m$. A-D, orginal magnification $\mathrm{x} 400$. An unspecific immunostaining of the acrosome of round spermatids is also shown.

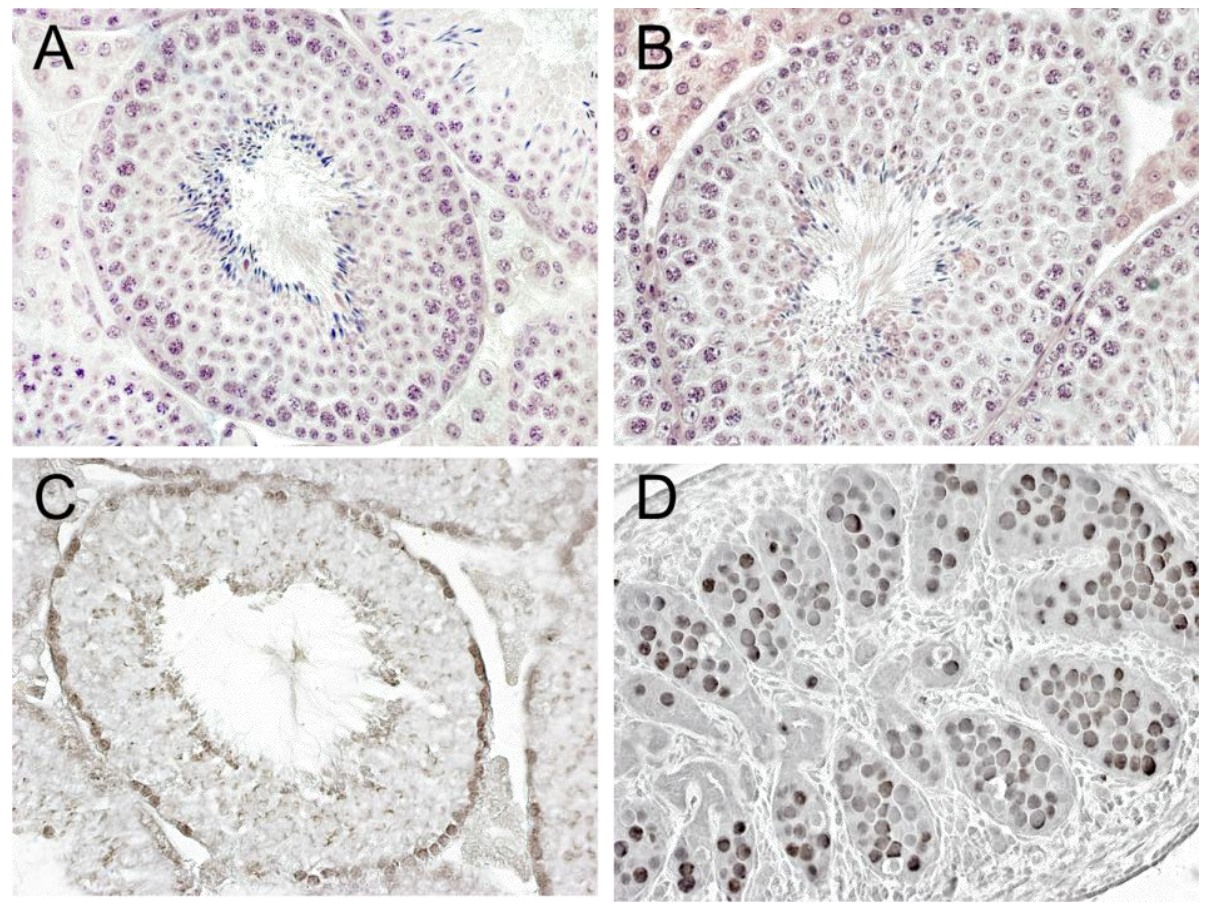

2.2.2. A suitable transgenic mouse model for functional analyses of TSPY in male fetal and adult germ cells

$T S P Y$ transgenic NMRI male mice of the line $T g(T S P Y) 9 J$ shm are phenotypically normal and fertile and spermatogenesis is neither impaired nor enhanced by the TSPY transgene (Figure 3) [47]. This is not surprising because it is likely that one or more as yet unknown gene(s) have taken over a TSPY 
analogous function within the mouse testis. One or more of the five known Tspy-like autosomal genes in the mouse genome could be candidates [49], and knock-out experiments performed on different autosomal Tspy-like genes would be a sensible attempt to elucidate their biological functions also within the testis. It is conceivable that spermatogenesis is not affected by the TSPY transgene due to the fact that the transgenic protein and its functional analog both fulfill the same functions within male germ cells, and thereby recognize the same partners. Hence, TSPY transgenic effects on germ cell development might become apparent when spermatogenesis proceeds under suboptimal conditions [47]. Schöner et al. [50] addressed the issue of whether TSPY is capable of restoring the

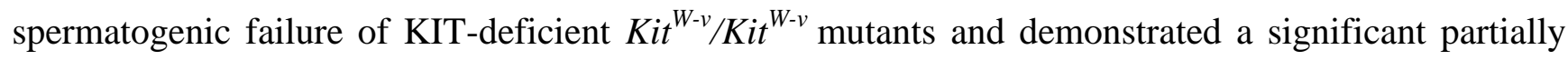
rescue of spermatogenesis, spermiogenesis and fertility in TSPY transgenic B6;NMRI-Kit ${ }^{W-v} / K_{i t}{ }^{W-v}$ male mice in comparison to controls (Figure 4).

Although these data point to a role of TSPY in fetal and adult germ cell proliferation, it is still unknown how TSPY supports the germ cell depleted spermatogenesis in the KIT deficient testis. The observed restoration of spermatogenesis could be due to a proliferative or apoptosis protective effect of the transgene in fetal or postnatal germ cell development. These findings suggest the line $T g(T S P Y) 9 J s h m$ as a suitable animal model for further studies to clarify TSPY putative functions in fetal and adult male germ cell development.

Figure 4. Histological haematoxylin and eosin stained sections of testes $(\mathbf{A}, \mathbf{B}$, orginal magnification $\mathrm{x} 200)$ and epididymides (C,D, orginal magnification $\mathrm{x} 400)$ of adult

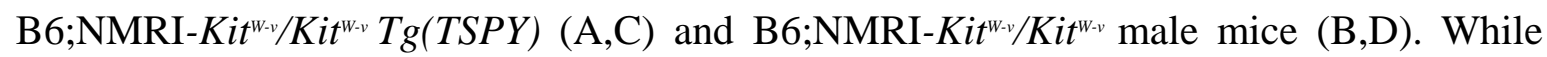
spermatogenesis arrests at the spermatogonial stage in the non transgenic B6;NMRI-Kit ${ }^{W-}$ $v / K^{W t^{W-v}}$ testis (B) and no sperm are present in the respective epididymis, a partial rescue of spermatogenesis and spermiogenesis is shown in the TSPY transgenic Kit-deficient testis (A). The respective epididymis is also filled with sperm (C).

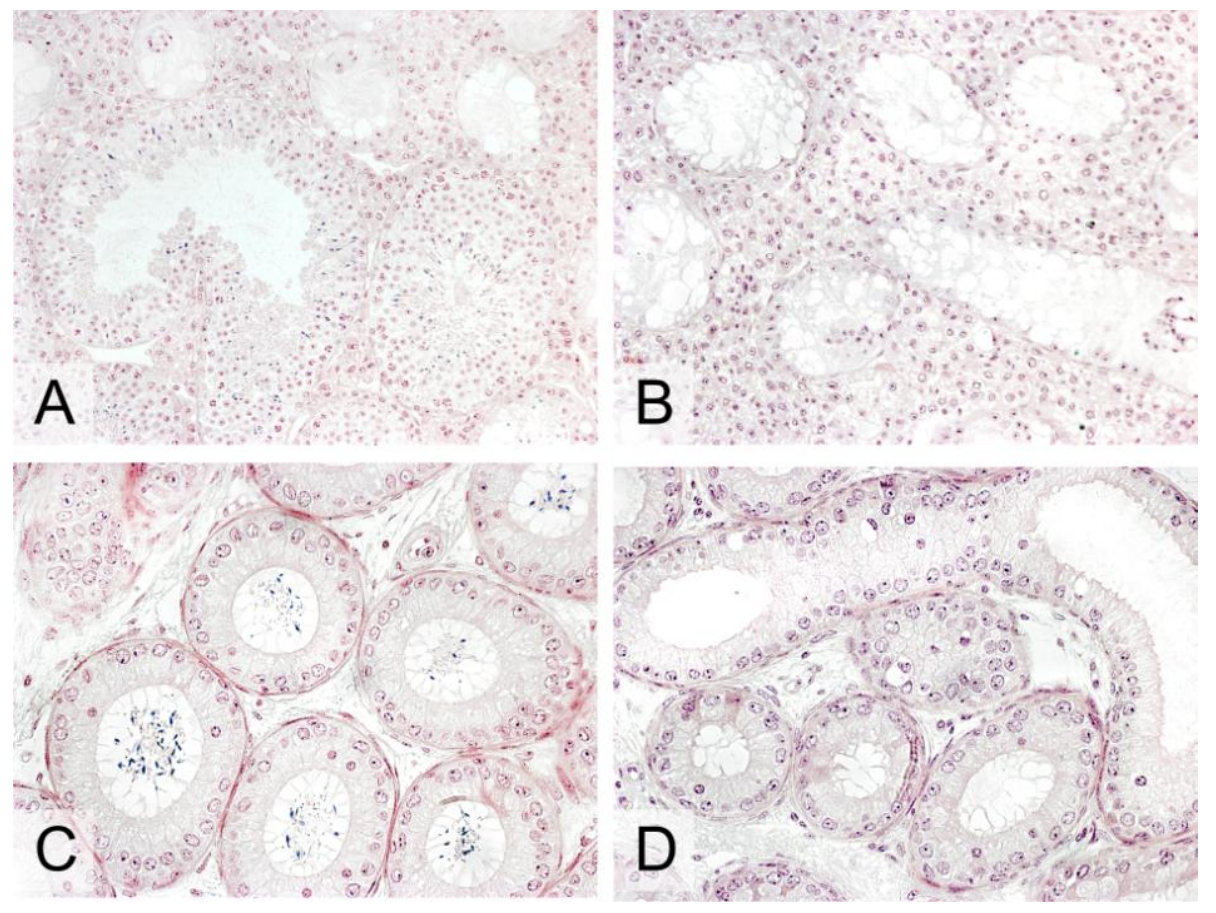


2.2.3. The line $T g(T S P Y) 9 J s h m$ is insufficient to elucidate TSPY role in gonadoblastoma formation and testicular tumorigenesis

It should be mentioned here that TSPY transgenic mice of the line $T g(T S P Y) 9 J \operatorname{shm}$ are not susceptible to any somatic or gonadal tumors [47,50,51]. The insusceptibility of the laboratory mouse for most testicular type-II germ cell tumors and their precursor stages questions the suitability of a TSPY transgenic mouse model as a tool to address TSPY putative oncogenic function in gonadal tumor formation. Testicular germ cell tumors in mice are nearly restricted to teratomas and embryonal carcinomas [40-42,57]. To the best of our knowledge only one transgenic mouse line that overexpressed the glia cell line derived neurotrophic factor (GDNF) promotes the formation of seminomatous tumors that mimics human seminomas [58]. Besides, both H-RasV12 transfected and cyclin D2 and cyclin E1 cotransfected mouse spermatogonial stem cells (SSCs) are capable of inducing placental alkaline phosphatase (PLAP) expressing germ cell tumors in recipient mouse testes [59]. On the other hand, it was demonstrated that a female founder harboring a $12.5 \mathrm{~kb}$ TSPY transgene, including also a complete $2.8 \mathrm{~kb}$ structural TSPY gene, developed gonadoblastoma-like structures in both ovaries, which expressed the TSPY transgene in a pattern resembling the expression pattern of TSPY in human gonadoblastoma [49,53]. In humans, gonadoblastoma arises in undifferentiated gondal tissue within dysgenetic gonads of intersex patients or phenotypic females carrying at least a part of Y chromosome that includes the GBY locus with an estimated risk of up to $30 \%$ [29,60], and human TSPY is at the moment the most likely candidate gene for GBY. The interesting finding that TSPY is able to induce gonadoblastoma-like structures in gonads of a female $T S P Y$ transgenic mouse [49,53] would indeed strengthen TSPY candidacy for GBY. As the TSPY transgenic line $\operatorname{Tg}(T S P Y) 9 \mathrm{~J} s \mathrm{hm}$ would never transmit the transgene to a female mice, the generation of a second TSPY transgenic line, with an autosomal or X-chromosomal integration site, that allows a proper expression of the TSPY transgene in the female gonad was one of our goals.

2.3. Transgenic lines $\mathrm{Tg}(\mathrm{TSPY}$-cre)33aYfcl and Tg(TSPY-cre)33bYfcl: A Cre-recombinase transgene under direction of a $2.4 \mathrm{~kb}$ TSPY promoter fragment

2.3.1. $2.4 \mathrm{~kb}$ human $T S P Y$ promoter fragment is insufficient to mediate a proper tissue-specific expression pattern of a reporter gene in transgenic mice

The first attempt to prove that a TSPY promoter fragment can be active in a female gonad was published in 2005 by Kido and Lau [16]. Two founder mice (one male and one female, both of B6CBAF1 origin) were generated by microinjection of a Cre-recombinase transgene driven by a 2383 bp putative TSPY promoter region and $43 \mathrm{bp}$ from the transcription start site of a human TSPY construct that was derived from the plasmid, pTSPY12.5 [7]. Only the female founder transcribed and transmitted the transgene properly, and generated - due to two different autosomal integration sitestwo different Cre recombinase transgenic lines with different transgenic copy numbers, designated as $T g$ (TSPY-cre)33aYfcl (42 copies) and Tg(TSPY-cre)33bYfcl (six copies). Both lines showed a similar tissue specific expression pattern but differed in expression strength of the Cre-recombinase transgene. Due to higher expression levels of the transgene in the line Tg(TSPY-cre)33aYfcl, this line was characterized in much more detail and will be discussed in this review. 2383 bp putative TSPY 
promoter region induced transgenic transcripts prominently in the gonads (testes and ovaries) and the brain (cerebral cortex and cerebellum) and to a lesser extent in the spleen but not in other organs, such as seminal vesicle, prostate, lung, heart, liver, kidney, thymus and uterus (Table1; [16]). Especially the expression of the Cre-recombinase transgene in the brain (cerebellum and cerebral cortex) of male and female mice of the line $T g(T S P Y$-cre $) 33 a Y f c l$ [16] and the detection of human TSPY transgenic transcripts in the brain (cerebrum) of mice of the line Tg(TSPY)9Jshm [47] are both striking findings, and the availability of TSPY ESTs derived from the medulla of human brains (BX281192, B1828033) in databases [16] could point to an up to now unexpected role of TSPY in the male brain. Because the $T S P Y$-Cre-recombinase transgene expression pattern mirrors widely that of the human ortholog, $2.4 \mathrm{~kb}$ of the putative $T S P Y$ promoter seem to be sufficient to direct a proper tissue-specific expression pattern of a TSPY-Cre recombinase transgene.

2.3.2. $2.4 \mathrm{~kb}$ promoter of human $T S P Y$ is sufficient to direct the expression of a reporter gene in oocytes but failed to induce a proper testicular expression pattern

Double transgenic TSPY-Cre;Z/EG mice were generated by crossing mice of the line $T g$ (TSPY-cre)33aYfcl with a Z/EG reporter line [61]. These TSPY-Cre;Z/EG mice expressed EGFP specifically after Cre recombinase-mediated action. The $2.4 \mathrm{~kb}$ fragment of the human TSPY promoter was capable of producing green fluorescence in testicular (round and elongated spermatids) and ovarian (oocytes of late primary and secondary follicles) germ cells (Figure 5) and in neurons of the central and peripheral nervous system in adult double transgenic mice [16].

Figure 5. Expression of EGFP in testis (A) and ovary (B) of double transgenic TSPY-Cre;Z/EG mice [16]. DAPI (blue) counterstained the DNA and EGFP fluorescence is shown in green. Within the testis (A), EGFP is exclusively expressed in round and elongated spermatids (stained in green), but not in other germ cells or somatic cells within the testis (stained blue). In B, an immunofluorescence image of an ovary from an 8-day old TSPY-Cre;Z/EG mice is shown. EGFP is expressed exclusively in maturing oocytes (stained green), while granulosa cells within the ovary were EGFP negative (stained blue). Figure 5 is published with the acknowledgement of Tatsuo Kido as the source.
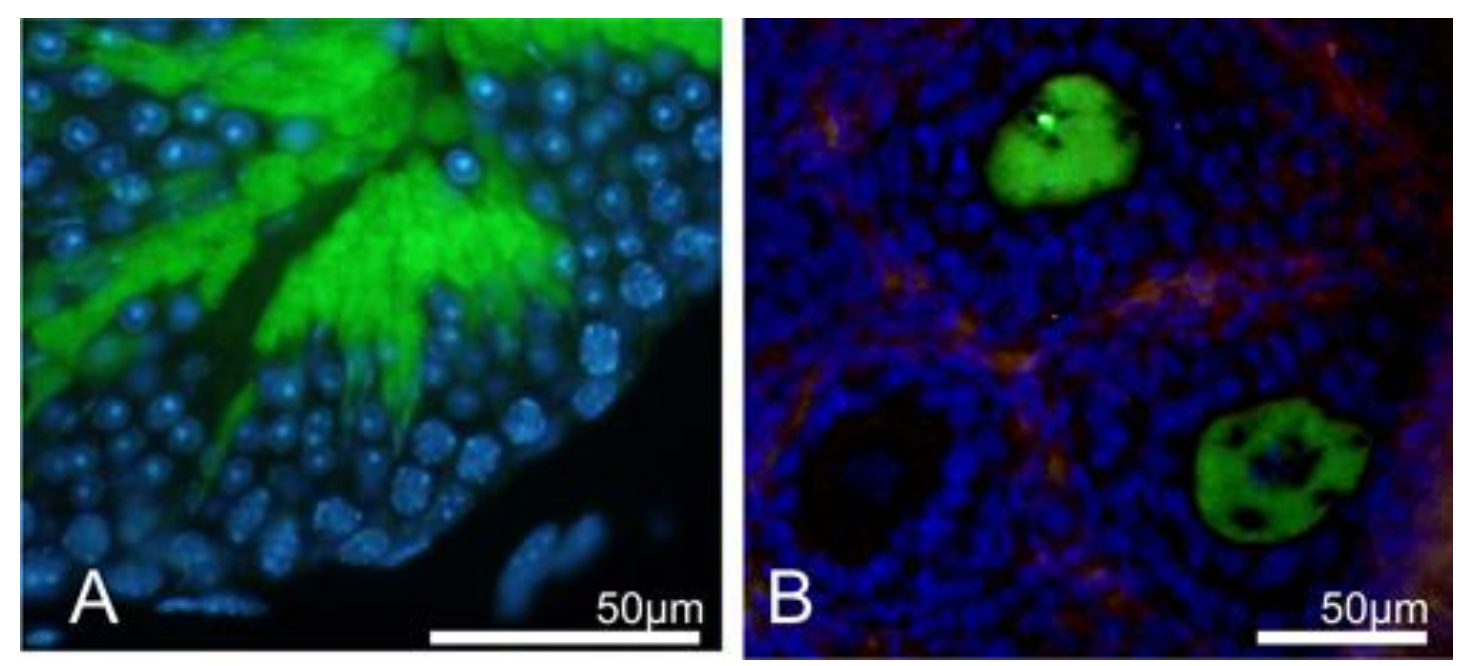
A remarkable finding of this study is that a $2.4 \mathrm{~kb}$ promoter of human TSPY is sufficient to direct the expression of a reporter gene in ovarian oocytes. Cre-recombinase transcripts were detected in ovaries of the embryonal stage E12.5, of newborn and in ovaries of prepubertal transgenic $T g$ (TSPY-cre)33aYfcl females but EGFP expression in double transgenic mice was not observed in primary oocytes, suggesting that the used promoter fragment is more active at late meiotic stages [16]. The activity of a $2.4 \mathrm{~kb}$ human TSPY promoter in an ovarian environment is of great importance, and supports the hypothesis that TSPY is indeed GBY [16,53].

The aberrant testicular expression pattern of adult double transgenic mice (Figure 5) in comparison to that observed in humans and in transgenic males of the line $T g(T S P Y) 9 J \operatorname{shm}$ is astonishing, and could partially be due to spermatogonial and spermatocytic enhancer elements that are present within the first 540 nucleotides at the $5^{\prime}$ end of the used 2923 bp putative TSPY promoter region in line $T g(T S P Y) 9 J s h m$ [47]. Further TSPY promoter analyses are indispensable to confirm this assumption. It was also shown by RT-PCR analyses that Cre transgenic transcripts are present in embryonal stage E12.5, newborn and subadult transgenic mice testes at time points when germ cells have not yet undergone second meiotic division [16]. These observations point to an insufficient expression level of the transgene in early spermatogenic germ cells. It is noteworthy that the $2.4 \mathrm{~kb} T S P Y$ promoter fragment also directed expression of the reporter gene in elongated spermatids similar to the testicular expression pattern of rat Tspy [62], and it is conceivable that the mouse preserved still parts of the rodent Tspy expression pattern by expressing the Cre-recombinase transgene preferentially in spermatids. On the other hand, the findings that TSPY is not expressed in elongated spermatids in humans [15,16] and in transgenic mice of the line Tg(TSPY)9Jshm [47] do not support the notion that laboratory mouse perpetuates the testicular expression pattern of rat Tspy. As both lines, $T g(T S P Y$-cre)33aYfcl and $T g(T S P Y$-cre)33bYfcl, with the transgene integrated at different chromosomal site, do not differ with respect to their EGFP testicular expression pattern, the influence of a chromosomal position effect on testicular expression can be excluded [46]. Finally, it can also not be ruled out that the observed differences in testicular expression in the transgenic lines $T g$ (TSPY-cre)33aYfcl, Tg(TSPY-cre)33bYfcl and Tg(TSPY)9Jshm could also be due to genetic background differences of the used mouse strains. Fertility of the EGFP expressing male and female double transgenic mice was normal and not affected by the presence of either transgene.

An intriguing finding of the adult TSPY-Cre;Z/EG mice was the neuron specific EGFP expression in brain, including the cerebellum, cerebral cortex and hippocampus. It is also noteworthy that EGFP was expressed in the optic cord and the trigeminal nerve of the brain stem but could not be detected in the anterior lobe of the pituitary gland in adult mice [16]. At embryonal stages E12.5-13.5, EGFP is specifically expressed in the trigeminal ganglia, the trigeminal nerve and in neurons of the dorsal root ganglia. These findings raise the question whether the TSPY promoter is also active in human neurons, and whether TSPY is able to fulfill yet unknown function in neural development and within the brain.

\section{Conclusions and outlook}

The functions of human TSPY genes are still unknown, but multiple in vivo and in vitro studies point to a role of TSPY in cell cycle regulation of fetal and spermatogenic germ cells. Especially the multiplicity and sequence diversity of human TSPY gene copies make the analyses of regulation, 
expression and function extremely difficult. The identification of a non- functional murine single-copy Tspy-ps pseudogene only in species of the subgenus $M u s$, including the laboratory mouse, enabled the generation of different transgenic mouse models as in vivo models for functional, regulative and expression analyses of human TSPY. Despite multiple efforts by several groups, only three classical transgenic mouse models have been successfully established, although much more founding transgenic mice have been generated. Overall, 10 founder mice were generated, harboring a complete $2.8 \mathrm{~kb}$ structural TSPY gene and 2.4 or $2.9 \mathrm{~kb} T S P Y$ promoter region, but only one male founder transmitted the transgene with paternal inheritance pattern [47,49]. It indeed seems that TSPY transgenic mice are difficult to establish [49], and it is nevertheless tempting to speculate that a Y chromosomal transgenic integration is essential for a successful transgenic mouse model. It is also conceivable that TSPY transgenes with autosomal or X-chromosomal integration site could be dysregulated, and that an ectopic expression of TSPY in other organs than the testis and prostate is harmful for the host [49]. The mouse Tspy-ps pseudogene could be used in future experiments as a targeting site to "smuggle" a human TSPY gene under the dependence of a tissue specific promoter on the mouse Y chromosome. A comparable targeting strategy for a Y-specific integration of a transgene was already successfully established in 2005 [63]. A murine myostatin (MSTN) coding sequence under the control of a rat myosin light chain $1 \mathrm{~F}(M L C 1 F)$ promoter and 1/3 enhancer for expression in skeletal muscle was introduced on the mouse Y chromosome in gene-targeted mice [63].

With the help of three transgenic mouse lines harboring reporter genes or a complete human TSPY gene driven by $1.3 \mathrm{~kb}$ [43], $2.4 \mathrm{~kb}$ [16] and $2.9 \mathrm{~kb}$ [47] of the TSPY promoter, it was shown that $2.9 \mathrm{~kb}$ of the human TSPY promoter is sufficient to direct a proper testis specific expression pattern of a transgene in transgenic mice. Especially the latter model can serve as an animal model to contribute significantly to our understanding of TSPY regulation, expression and function, and could be useful to address TSPY putative oncogenic role in prostatic and testicular tumor formation in future studies. Besides the transgenic mouse line TSPY-TAg23 represents a useful tumor mouse model for pituitary adenomas secreting PRL and ACTH [43]. The different testicular expression pattern of the transgenic lines $T g$ (TSPY-cre)33aYfcl, Tg(TSPY-cre)33bYfcl and Tg(TSPY)9Jshm (Table 1) could in general be influenced by the different length of the promoter, the integration sites of the transgenes within the mouse genome, and the genetic background of the hosts [49]. As only one TSPY transgenic mouse line has been successfully established, the generation of further TSPY transgenic lines with Y-chromosomal or autosomal integration sites must be a desirable main aim. Especially the interesting finding that TSPY is able to induce gonadoblastoma-like structures in gonads of a female TSPY transgenic mouse supports the hypothesis that TSPY could be indeed GBY. Generation of a second TSPY transgenic line, with an autosomal or X-chromosomal integration site, that allows a proper expression of the TSPY transgene in ovaries should be a main aim of future studies.

As the genetic background of a mouse model can dramatically impact on its phenotype [34,50,51], the choice of the proper mouse strain for transgenesis is important to avoid the pitfall of time-consuming backcrossing into the genetic background of choice.

All these findings raise the question, whether alternative animal models could be utilized in future studies aimed at investigating regulation, expression and function of TSPY. As the testicular expression pattern of rat Tspy does not properly mimic that of human TSPY, a targeted gene knock-out of rat Tspy would only partially be useful to uncover the TSPY function in humans. It is supposed that 
rat Tspy could act as histone chaperone in elongated spermatids, by the time core histones are replaced by basic protamines [62]. The fact that rat and human TSPY can both interact in vitro with core histones suggest that both direct similar functions in spermiogenesis [62]. Especially the recent developed zinc finger nuclease technology which was also successfully applied to the rat $[64,65]$ could be suitable for the generation of targeted $r$ Tspy gene disruption. The zinc finger nuclease technology seems to be in general a promising tool to generate knock-out mutations at specific loci in mammalians and will offer new opportunities for Tspy gene knock-outs in other rodent species. Finally knock-out experiments performed on each of the six different Tspy-like genes would be a feasible approach to elucidate their biological functions also within the testis.

\section{Acknowledgements}

We thank Melanie Scholz of the Institute of Human Genetics, Hannover Medical School, Alexandra Kotsiari, Center for Addiction Research, Department of Psychiatry, Social Psychiatry and Psychotherapy, Hannover Medical School, Hannover and Frank Dechend of the Laboratory of Cytogenetic and Molecular Genetics in Hildesheim for technical assistance. We are indebted to Tatsuo Kido, Division of Cell and Developmental Genetics, Department of Medicine, VA Medical Center, University of California, San Francisco for contributing Figure 5 and giving advice in the interpretation of the presented results.

\section{References and Notes}

1. Skaletsky, H.; Kuroda-Kawaguchi, T.; Minx, P.J.; Cordum, H.S.; Hillier, L.; Brown, L.G.; Repping, S.; Pyntikova, T.; Ali, J.; Bieri, T.; Chinwalla, A.; Delehaunty, A.; Delehaunty, K.; Du, H.; Fewell, G.; Fulton, L.; Fulton, R.; Graves, T.; Hou, S.F.; Latrielle, P.; Leonard, S.; Mardis, E.; Maupin, R.; McPherson, J.; Miner, T.; Nash, W.; Nguyen, C.; Ozersky, P.; Pepin, K.; Rock, S.; Rohlfing, T.; Scott, K.; Schultz, B.; Strong, C.; Tin-Wollam, A.; Yang, S.P.; Waterston, R.H.; Wilson, R.K.; Rozen, S.; Page DC. The male-specific region of the human Y chromosome is a mosaic of discrete sequence classes. Nature 2003, 423, 825-837.

2. Manz, E.; Schnieders, F.; Muller Brechlin, A.; Schmidtke, J. TSPY-related sequences represent a microheterogenous gene family organized as constitutive elements in DYZ5 tandem repeat units on the human Y chromosome. Genomics 1993, 17, 726-731.

3. Dechend, F.; Williams, G.; Skawran, B.; Schubert, S.; Krawczak, M.; Tyler-Smith, C.; Schmidtke, J. TSPY variants in six loci on the human Y chromosome. Cytogenet. Cell Genet. 2000, 91, 67-71.

4. Graves, M.J.A. The rise and fall of Sry. Trends Genet. 2002, 18, 259-264.

5. Vogel, T.; Schmidtke, J. Structure and function of TSPY, the Y-chromosome gene coding for the "testis-specific protein". Cytogenet. Cell Genet. 1998, 80, 209-213.

6. Kim, H.S.; Hirai, H.; Takenaka, O. Molecular features of the TSPY gene of gibbons and Old World monkeys. Chromosome Res. 1996, 4, 500-506.

7. Zhang, J.S.; Yang-Feng, T.L.; Muller, U.; Mohandas, T.K.; de Jong, P.J.; Lau, Y.F. Molecular isolation and characterization of an expressed gene from the human Y chromosome. Hum. Mol. Genet. 1992, 1, 717-726. 
8. Jakubiczka, S.; Schnieders, F.; Schmidtke, J. A bovine homologue of the human TSPY gene. Genomics 1993, 17, 732-735.

9. Verkaar, E.L.; Zijlstra, C.; van’t Veld, E.M.; Boutaga, K.; van Boxtel, D.C; Lenstra, J.A. Organization and concerted evolution of the ampliconic Y-chromosomal TSPY genes from cattle. Genomics 2004, 84, 468-474.

10. Repping, S.; van Daalen, S.K.M.; Brown, L.G.; Korver, C.M.; Lange, J.; Marszalek, J.D.; Pyntikova, T.; van der Veen, F.; Skaletsky, H.; Page, D.C.; Rozen, S. High mutation rates have driven extensive structural polymorphism among human Y chromosome. Nat. Genet. 2006, 38, 463-467.

11. Mazeyrat, S.; Mitchell, M.J. Rodent Y chromosome TSPY gene is functional in rat and nonfunctional in mouse. Hum. Mol. Genet. 1998, 7, 557-562.

12. Schubert, S.; Dechend, F.; Skawran, B., Kunze, B.; Winking, H.; Weile, C.; Romer, I.; Henberger, M.; Fundele, R.; Sharma, T.; Schmidtke, J. Silencing of the Y-chromosomal gene tspy during murine evolution. Mamm. Genome 2000, 11, 288-291.

13. Karwacki, V.; Kovac, J.; Mauceri, G.; Backhaus, A.; Föhse, L.; Schmidtke, J.; Schubert, S. Tspy is non functional in the Mongolian gerbil but functional in the Syrian hamster. Genomics 2006, $88,65-73$.

14. Honecker, F.; Stoop, H.; de Krijger, R.R.; Lau, Y.F.C.; Bokemeyer, C.; Looijenga, L.H. Pathobiological implications of the expression of markers of testicular carcinoma in situ by fetal germ cells. J. Pathol. 2004, 203, 849-857.

15. Schnieders, F.; Dork, T.; Arnemann, J.; Vogel, T.; Werner, M.; Schmidtke, J. Testis-specific protein, Y-encoded (TSPY) expression in testicular tissues. Hum. Mol. Genet. 1996, 5 1801-1807.

16. Kido, T.; Lau, Y.F.C. A Cre gene directed by a human TSPY promoter is specific for germ cells and neurons. Genesis 2005, 42, 263-275.

17. Lau, Y.F.C.; Lau, H.W.; Kömüves, L.G. Expression pattern of a gonadoblastoma candidate gene suggests a role of the Y chromosome in prostate cancer. Cytogenet. Genome Res. 2003, 101, 250-260.

18. She, X.; Rohl, C.A.; Castle, J.C.; Kulkarni, A.V.; Johnson, J.M.; Chen, R. Definition, conservation and epigenetics of housekeeping and tissue-enriched genes. BMC Genomics 2009, $10,269$.

19. Arnemann, J.; Epplen, J.T.; Cooke, H.J.; Sauermann, U.; Engel, W.; Schmidtke, J. A human Ychromosomal DNA sequence expressed in testicular tissue. Nucleic Acids Res. 1987, 15, 8713-8724.

20. Arnemann, J.; Jakubiczka, S.; Thüring, S.; Schmidtke, J. Cloning and sequence analysis of a human Y-chromosome-derived, testicular cDNA, TSPY. Genomics 1991, 11, 108-114.

21. Oram, S.W.; Liu, X.X.; Lee, T.L.; Chan, W.Y.; Lau, Y.F.C. TSPY potentiates cell proliferation and tumorigenesis by promoting cell cycle progression in HeLa and NIH3T3 cells. BMC Cancer 2006, 6, 154.

22. Li, Y.; Lau, Y.F.C. TSPY and its X-encoded homologue interact with cyclin B but exert contrasting functions on cyclin-dependent kinase 1 activities. Oncogene 2008, 27, 6141-6150. 
23. Kido, T.; Lau, Y.F.C. The human Y-encoded testis-specific protein interacts functionally with eukaryotic translation elongation factor eEF1A, a putative oncoprotein. Int. J. Cancer 2008, 123, 1573-1585.

24. Lau, Y.F.C.; Chou, P.M.; Iezzoni, J.C.; Alonzo, J.A.; Kömüves, L.G. Expression of a candidate gene for the gonadoblastoma locus in gonadoblastoma and testicular seminoma. Cytogenet. Cell Genet. 2000, 91, 160-164.

25. Honecker, F.; Stoop, H.; Mayer, F.; Bokemeyer, C.; Castrillon, D.H.; Lau, Y.F.C.; Looijenga, L.H.J.; Oosterhuis, J.W. Germ cell lineage differentiation in non-seminomatous germ cell tumors. J. Pathol. 2006, 208, 395-400.

26. Li, Y., Tabatabai, L.; Lee, T-L.; Hatakeyama, S.; Ohyama, C.; Chan, W-Y.; Looijenga, L.H.J.; Lau, Y.F.C. The Y-encoded TSPY protein: a significant marker potentially plays a role in the pathogenesis of testicular germ cell tumors. Hum. Pathol. 2007, 38, 1470-1481.

27. Hildenbrand, R.; Schröder, W.; Brude, E.; Schepler, A.; König, R.; Stutte, H.J.; Arnemann J. Detection of TSPY protein in a unilateral microscopic gonadoblastoma of a Turner mosaic patient with a Y-derived marker chromosome. J. Pathol. 1999, 189, 623-626.

28. Kersemaekers, A.M.F.; Honecker, F.; Stoop, H.; Cools, M.; Molier, M.; Wolffenbuttel, K.; Bokemeyer, C.; Li, Y.; Lau, Y.F.C.; Oosterhuis, J.W.; Looijenga, L.H.J. Identification of germ cells at risk for neoplastic transformation in gonadoblastoma. An immunohistochemical study for Oct3/4 and TSPY. Hum. Pathol. 2005, 36, 512-521.

29. Li, Y.; Vilain, E. ; Conte, F. ; Rajpert-De Meyts, E. ; Lau, Y.FC. Testis-specific protein Y encoded gene is expressed in early and late stages of gonadoblastoma and testicular carcinoma in situ. Urol. Oncol. Seminars of Original Investigations 2007, 25,141-146.

30. Page DC. Hypothesis: a Y-chromosomal gene causes gonadoblastoma in dysgenetic gonads. Development 1987, 101, 151-155.

31. Yin, Y.H.; Li, Y.Y.; Qiao, H.; Wang, H.C.; Yang, X.A.; Zhang, H.G.; Pang, X.W.; Zhang, Y.; Chen, W.F. TSPY is a cancer testis antigen expressed in human hepatocellular carcinoma. $B r . J$. Cancer 2005, 93, 458-463.

32. Gallagher, W.M.; Bergin, O.E.; Rafferty, M.; Kelly, Z.D.; Nolan, I.M.; Fox, E.J.; Culhane, A.C.; McArdle, L.; Fraga, M.F.; Hughes, L.; Currid, C.A.; O'Mahony, F.; Byrne, A.; Murphy, A.A.; Moss, C.; McDonnell, S., Stallings, R.L.; Plumb, J.A.; Esteller, M.; Brown, R.; Dervan, P.A.; Easty, D.J. Multiple markers for melanoma progression regulated by DNA methylation: insights from transcriptomic studies. Carcinogenesis 2005, 26, 185618-185667.

33. Gordon, J.W.; Scangos, G.A; Plotkin, D.J.; Barbosa, J.A.; Ruddle, F.H. Genetic transformation of mouse embryos by microinjection of purified DNA. Proc. Natl. Acad. Sci. U. S. A. 1980, 77, 7380-7384.

34. Castrop, H. Genetically modified mice-successes and failures of a widely used technology. Eur. J. Physiol. 2010, 459, 557-567.

35. Vogel, T.; Boettger-Tong, H.; Nanda, I.; Dechend, F.; Agulnik, A.I.; Bishop, C.E.; Schmid, M.; Schmidtke, J. A murine TSPY. Chromosome Res. 1998, 6, 35-40.

36. Hanahan, D. Heritable formation of pancreatic beta-cell tumours in transgenic mice expressing recombinant insulin/simian virus 40 oncogenes. Nature 1985, 315, 115-22. 
37. Garabedian, E.M.; Humphrey, P.A.; Gordon, J.I. A transgenic mouse model of metastatic prostate cancer originating from neuroendocrine cells. Proc. Natl. Acad. Sci. U. S. A. 1998, 95, 15382-15387.

38. Rathi, A.V.; Sáenz Robles, M.T.; Cantalupo, P.G.; Whitehead, R.H.; Pipas, J.M. Simian virus 40 $\mathrm{T}$-antigen-mediated gene regulation in enterocytes is controlled primarily by the Rb-E2F pathway. J. Virol. 2009, 83, 9521-9531.

39. Jeet, V.; Russell, P.J.; Khatri, A. Modeling prostate cancer: a perspective on transgenic mouse models. Cancer Metastasis Rev. 2010, 29, 123-142.

40. Oosterhuis, J.W.; Looijenga, H.J. Testicular germ-cell tumors in a broader perspective. Nature Reviews 2005, 5, 210-222.

41. Stevens, L.C.; Little, C.C. Spontaneous Testicular Teratomas in an Inbred Strain of Mice. Proc. Natl. Acad. Sci. U. S. A. 1954, 40, 1080-1087.

42. Stevens, L.C. Teratocarcinogenesis and spontaneous parthenogenesis in mice. Results Probl. Cell Differ. 1980, 11, 265-274.

43. Tascou, S.; Trappe, R.; Nayernia, K.; Jarry, H.; König, F.; Schulz-Schaeffer, W.; Saeger, W.; Meinhardt, A.; Engel, W.; Schmidtke, J.; Burfeind, P. TSPY-LTA transgenic mice develop endocrine tumors of the pituitary and adrenal gland. Mol. Cell Endocrinol. 2003, 200, 9-18.

44. Tascou, S.; Nayernia, K.; Samani, A.; Schmidtke, J.; Vogel, T.; Engel, W.; Burfeind, P. Immortalization of murine male germ cells at a discrete stage of differentiation by a novel directed promoter-based selection strategy. Biol. Reprod. 2000, 63, 1555-1561.

45. Barausse, M.; Attanasio, R.; Dallabonzana, D.; Oppizzi, G.; Veronese, S.; Lasio, G.; Valentini, L.G.; Cozzi, R. From macroprolactinoma to concomitant ACTH-PRL hypersecretion with Cushing's disease. J. Endocrinol Invest. 2000, 23, 107-111.

46. Clark, A.J.; Bissinger, P.; Bullock, D.W.; Damak, S.; Wallace, R.; Whitelaw, C.B.; Yull, F. Chromosomal position effects and the modulation of transgene expression. Reprod. Fertil. Dev. 1994, 6, 589-598.

47. Schubert, S.; Skawran, B.; Dechend, F.; Nayernia, K.; Meinhardt, A.; Nanda, I.; Schmid, M.; Engel, W.; Schmidtke, J. Generation and characterization of a transgenic mouse with a functional human TSPY. Biol. Reprod. 2003, 69, 968-975.

48. Dechend, F. Hannover Medical School, Hannover, Germany. Personal communication, 2000.

49. Lau, Y.F.; Li, Y.; Kido, T. Gonadoblastoma locus and the TSPY gene on the human Y chromosome. Birth Defects Res C Embryo Today. 2009, 87, 114-122.

50. Schöner, A.; Adham, I.; Mauceri, G.; Marohn, B.; Vaske, B.; Schmidtke, J.; Schubert, S. Partial Rescue of the KIT-Deficient Testicular Phenotype in KitW-v/KitW-v Tg(TSPY)9 Mice. Biol. Reprod. 2010, 83, 20-26

51. Schubert, S.; Kamino, K.; Böhm, D.; Adham, I.; Engel, W.; von Wasielewski, R.; MoharreghKhiabani, D.; Mauceri, G.; Vaske, B.; Meinhardt, A.; Schöner, A.; Gonzalez-Fassrainer, D.; Schmidtke, J. TSPY expression is variably altered in transgenic mice with testicular feminization. Biol. Reprod. 2008, 79, 125-133.

52. Schubert, S. Hannover Medical School, Hannover, Germany. Unpublished work, 2010.

53. Lau, Y.F.; Li, Y.; Kido, T. Role of the Y located putative gonadoblastoma gene in human spermatogenesis. Syst. Biol. Reproduc. Med., in press. 
54. Lau, Y.F.C. chromosome genetics' 99 Gonadoblastoma, testicular and prostate cancers, and the TSPY gene. Am. J. Hum. Genet. 1999, 64, 921-927.

55. Lau, Y.F.C; Zhang, J. Expression analysis of thirty-one Y chromosome genes in human prostate cancer. Mol. Carcin. 2000, 27, 308-321.

56. Singh, A.P.; Bafna, S.; Chaudhary, K.; Venkatraman. G.; Smith, L.; Eudy, J.D.; Johansson, S.L.; Lin, M.F.; Batra, S.K. Genome-wide expression profiling reveals transcriptomic variation and perturbed gene networks in androgen-dependent and androgen-independent prostate cancer cells. Cancer Lett. 2008, 259, 28-38.

57. Gidekel, S.; Pizov, G.; Bergman, Y.; Pikarsky, E. Oct-3/4 is a dose-dependent oncogenic fate determinant. Cancer Cell. 2003, 4, 361-370.

58. Sariola, H.; Meng, X. GDNF-induced seminomatous tumours in mouse--an experimental model for human seminomas? APMIS. 2003, 111, 192-196.

59. Lee, J.; Kanatsu-Shinohara, M.; Morimoto, H.; Kazuki, Y.; Takashima, S.; Oshimura, M.; Toyokuni, S.; Shinohara, T. Genetic reconstruction of mouse spermatogonial stem cell selfrenewal in vitro by Ras-cyclin D2 activation. Cell Stem Cell. 2009, 5, 76-86.

60. Cools, M.; Stoop, H.; Kersemaekers, A.M.F.; Drop, S.L.S.; Wolffenbuttel, K.P.; Bourguignon, JP.; Slowikowska-Hilczer, J.; Kula, K.; Faratz, S.; Oosterhuis, J.W.; Looijenga, L.H.J. Gonadoblastoma arising in undifferentiated gonadal tissue within dysgenetic gonads. Clin. Endocrinol. Metab. 2005, 90, 5295-5303.

61. Novak, A.; Guo, C.; Yang, W.; Nagy, A.; Lobe, C.G. Z/EG, a double reporter mouse line that expresses enhanced green fluorescent protein upon Cre-mediated excision. Genesis 2000, 28, 147-155.

62. Kido, T.; Lau, Y.F. The rat Tspy is preferentially expressed in elongated spermatids and interacts with the core histones. Biochem. Biophys. Res. Commun. 2006, 350, 56-67.

63. Pirottin, D.; Grobet, L.; Adamantidis, A.; Farnir, F.; Herens, C.; Daa Schrøder, H.; Georges, M. Transgenic engineering of male-specific muscular hypertrophy. Proc. Natl. Acad. Sci. U. S. A. 2005, 102, 6413-6418.

64. Geurts, A.M.; Cost, G.J.; Freyvert, Y.; Zeitler, B.; Miller, J.C.; Choi, V.M.; Jenkins, S.S.; Wood, A.; Cui, X.; Meng, X.; Vincent, A.; Lam, S.; Michalkiewicz, M.; Schilling, R.; Foeckler, J.; Kalloway, S.; Weiler, H.; Ménoret, S.; Anegon, I.; Davis, G.D.; Zhang, L.; Rebar, E.J.; Gregory, P.D.; Urnov, F.D.; Jacob, H.J.; Buelow, R. Knockout rats via embryo microinjection of zincfinger nucleases. Science. 2009, 325, 433.

65. Geurts, A.M.; Cost, G.J.; Rémy, S.; Cui, X.; Tesson, L.; Usal, C.; Ménoret, S.; Jacob, H.J.; Anegon, I.; Buelow, R. Generation of gene-specific mutated rats using zinc-finger nucleases. Methods Mol. Biol. 2010, 597, 211-225.

(C) 2010 by the authors; licensee MDPI, Basel, Switzerland. This article is an Open Access article distributed under the terms and conditions of the Creative Commons Attribution license (http://creativecommons.org/licenses/by/3.0/). 